\title{
Administration of Metallic Mercury by an Accidental Puncture in the Hand: A Case Report
}

\author{
Dilek Ersil Soysal ${ }^{\mathrm{a}, \mathrm{e}}$, Murat Tuzuner ${ }^{\mathrm{b}}$, Ali Riza Seren ${ }^{\mathrm{c}}$, Ebru Tekesin ${ }^{\mathrm{a}}$, Emrah Koc ${ }^{\mathrm{a}}$, \\ Yusuf Cem Kaplan ${ }^{\mathrm{d}}$, Sezin Hizar Turan ${ }^{\mathrm{a}}$, Sadi Bener ${ }^{\mathrm{d}}$, Mete Pekdiker ${ }^{\mathrm{a}}$, Cem Aslan ${ }^{\mathrm{b}}$
}

\begin{abstract}
Mercury is a heavy metal of known toxicity. Human toxicity varies with the form of mercury, the dose and the rate of exposure. Subcutaneous injection of elemental mercury is very rare and usually accidental. Diagnosis of mercury overload is difficult. The commonly used modalities (blood, urine, and/or hair levels) reflect recent exposure, and do not correlate with total body burden. We present a case of accidental subcutaneous administration of mercury in the hand and discuss its management.
\end{abstract}

Keywords: Mercury toxicity; Accidental

\section{Introduction}

Mercury is a naturally occurring metal that has elemental (metallic), inorganic, and organic forms. Elemental mercury is a shiny, silver-white liquid (quicksilver) obtained predominantly from the refining of mercuric sulfide in cinnabar ore [1]. Poisoning by metallic mercury is rare and usually accidental, including injury from a broken thermometer [2, 3]. The ill effects produced as a result of self administration of the metallic form of mercury have appeared in scattered case

Manuscript accepted for publication July 10, 2012

a Department of Internal Medicine, Izmir Katip Celebi University, Ataturk Research and Training Hospital, Izmir, Turkey

${ }^{\mathrm{b}}$ Department of Plastic, Reconstructive and Aesthetic Surgery, Izmir Katip Celebi University, Ataturk Research and Training Hospital, Izmir, Turkey

${ }^{\mathrm{c}}$ Department of Anesthesiology and Reanimation, Izmir Katip Celebi University, Ataturk Research and Training Hospital, Izmir, Turkey

d Department of Pharmacology, Izmir Katip Celebi University, Ataturk

Research and Training Hospital, Izmir, Turkey

${ }^{\mathrm{e}}$ Corresponding author: Dilek Ersil Soysal, Izmir Katip Celebi

University, Ataturk Research and Training Hospital, Basln Sitesi,

Izmir, Turkey. Email: dileksoysal@hotmail.com

doi: http://dx.doi.org/10.4021/jmc810w reports [3]. Mercury poisoning is usually misdiagnosed because of the insidious onset, nonspecific signs and symptoms and lack of knowledge within the medical profession [4]. Here, we present a case of accidental subcutaneous administration of mercury in the hand and discuss its management.

\section{Case Report}

A 32-year-old woman presented with administration of metallic mercury was hospitalized in our clinic on May 2011. A mercury thermometer was broken in her hand, and wounded it accidentally. The metallic mercury inside the thermometer scattered throughout the soft tissue of the left hand. She complained of pain and redness with edema around the wound on the palm.

Physical examination was unremarkable. Laboratory findings, such as complete blood cell count, urine analysis, serum levels of blood urea nitrogen, creatinine, electrolytes, calcium and phosphorus, and liver function tests were normal. Analysis of the arterial blood gas levels were within normal limits on room air. Chest radiograph, and ultrasound of the abdomen were normal. Anteroposterior and lateral radiographs of the left hand showed multipl scattered metal-density opacities localized in the soft tissues around the metacarpals and carpometacarpal joint (Fig. 1). Following her hospitalization, on the 17th May she was operated for removing the mercury and broken parts of the thermometer. Mercury could not be removed totally.

By atomic absorption method, blood mercury level was $14.1 \mu \mathrm{g} / \mathrm{L}(\mathrm{N}: 0-10 \mu \mathrm{g} / \mathrm{L})$, and 24-h urine mercury level was $12.0 \mu \mathrm{g} / \mathrm{d}(\mathrm{N}: 0-20 \mu \mathrm{g} / \mathrm{d})$ at admission on the 17 th of May 2011. Over a 12-month of follow-up, the patient did not show any general or central nervous system symptoms of mercury intoxication. During this period, blood and 24-h urine mercury levels were monitored (Table 1). On the $21 \mathrm{st}$ of September, then on the 27th of October the patient was operated in view of the elevated blood mercury levels and the potential severity of mercury intoxication if not properly treated [2]. In the operation, it was impossible to excise all tissues where mercury had scattered because of reserving and protecting important anatomical structures like tendons and 


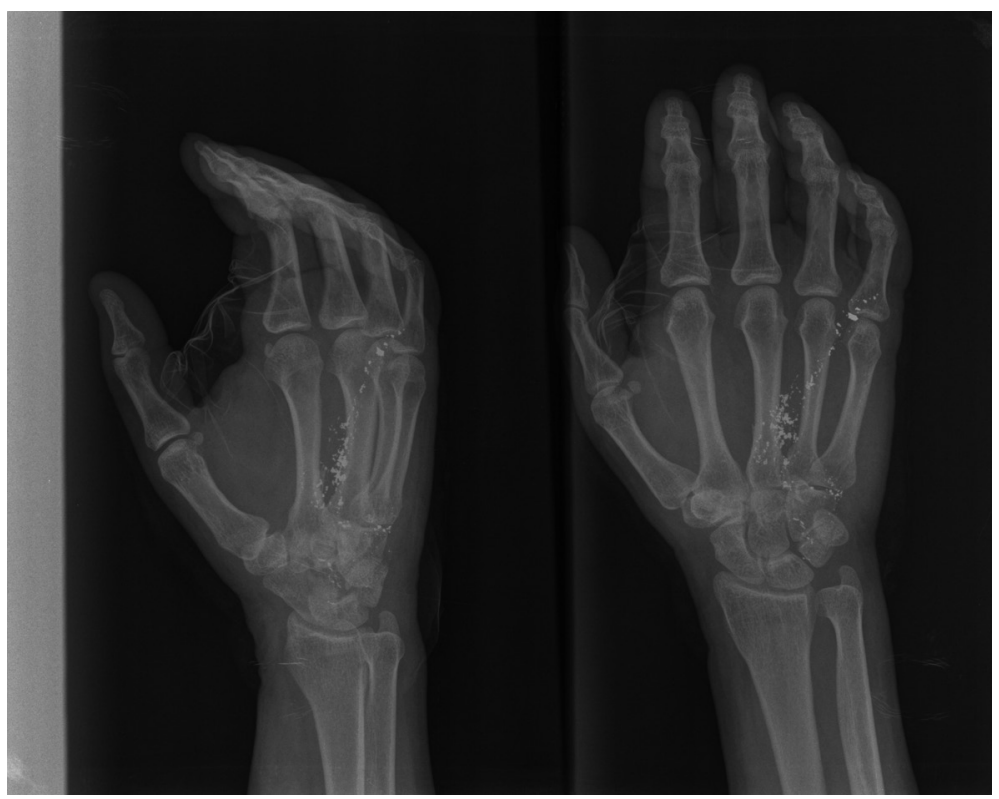

Figure 1. Preoperative lateral and anteroposterior radiographs of the left hand showing multipl scattered metaldensity opacities localized in the soft tissues around the metacarpus.

neurovascular tissues inorder to protect the hand function. Mainly residual mercury was left in the soft tissue around the carpometacarpal joint. X-ray scope used during the operation to evaluate the excision. Postoperative radiographs of the left hand showed residual metallic mercury was left in the soft tissues around the metacarpals and carpometacarpal joint (Fig. 2) and (Fig. 3). The histopathological examination of the removed tissue showed mercury as black pigmented globules and a foreign-body reaction with polymorphs, lymphocytes, histiocytes and fibrosis of chronic inflammation

Table 1. Blood and 24-h Urine Mercury Levels During Follow-Up of the Patient

\begin{tabular}{|c|c|c|}
\hline & $\begin{array}{l}\text { Mercury Level } \\
\text { (blood) } \mu \mathrm{g} / \mathrm{L}\end{array}$ & $\begin{array}{l}\text { Mercury Level } \\
\text { (24-h urine) } \mu \mathrm{g} / \mathrm{d}\end{array}$ \\
\hline 17.06 .2011 & 12.20 & 13.20 \\
\hline 31.06 .2011 & 14.30 & 23.30 \\
\hline 16.07.2011 & 24.00 & 33.20 \\
\hline 01.08.2011 & 9.80 & 25.00 \\
\hline 17.08.2011 & 10.80 & 35.00 \\
\hline 15.09 .2011 & 11.77 & 85.60 \\
\hline 17.10 .2011 & 19.60 & 125.00 \\
\hline 31.10 .2011 & 16.80 & 172.00 \\
\hline 21.01 .2012 & 9.70 & 58.00 \\
\hline 16.03 .2012 & 3.70 & 42.00 \\
\hline
\end{tabular}




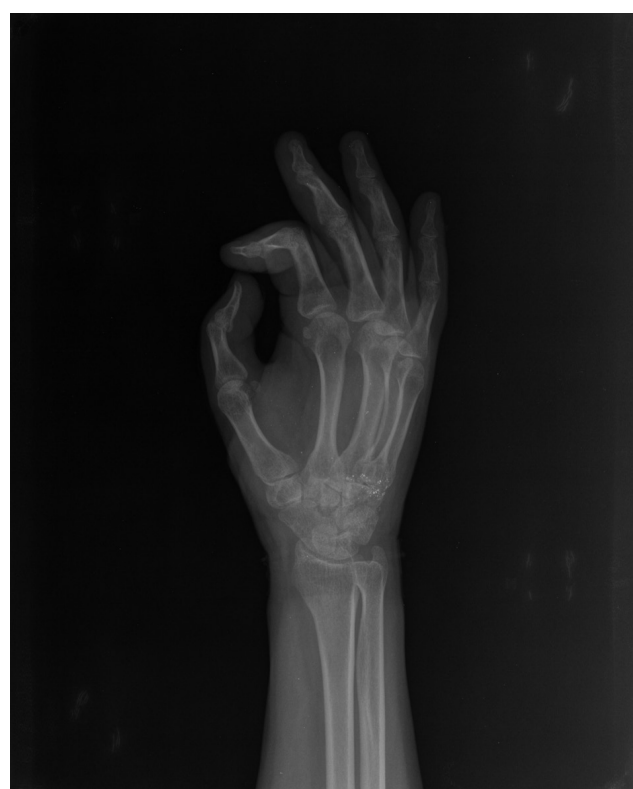

Figure 2. Postoperative lateral radiography of the left hand showing that multipl metallic mercury was left in the soft tissue around the metacarpus.

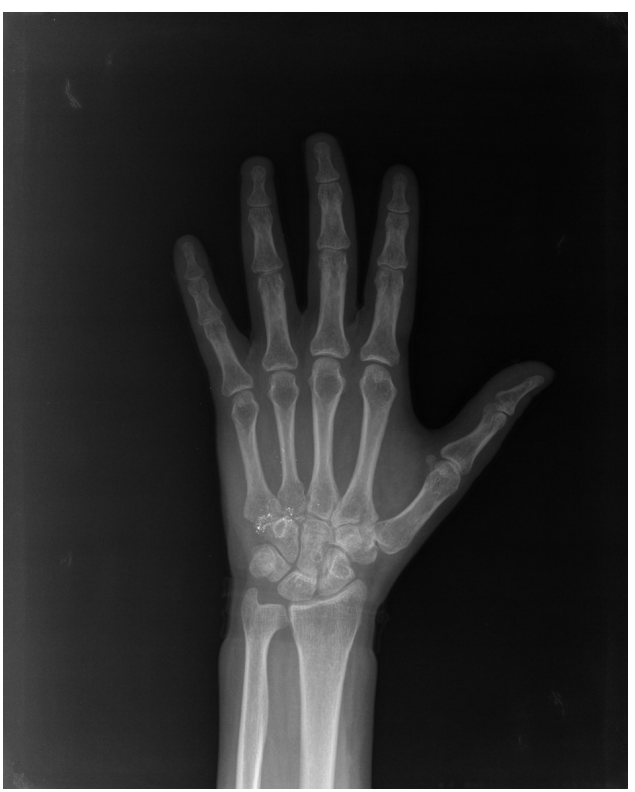

Figure 3. Postoperative anteroposterior radiography of the left hand showing that multipl metallic mercury was left in the soft tissue around the metacarpus. around the globules (Fig. 4a) and (Fig. 4b).

Clinical assessment of the patient and measurement of mercury levels in blood and urine were carried out. The patient presented with complaints of itching skin rash and painful abdomen after the third operation. Treatment with nonspecific agents such as low-sedating antihistamines and antacids had no effect on her symptoms. The symptoms regressed in time which was related to the gradual decrease of mercury concentrations in blood. None of the systemic symptoms and signs of mercury absorption were detected, and chelation therapy with dimercaprol was not required [5].
She has been followed for 12 months now.

\section{Discussion}

Mercury is still used in the manufacture of many technical and medical instruments, including sphygmomanometers, manometers, thermometers, and barometers [6]. Mercury in any form is toxic. Mercury poisoning can result from vapor inhalation, ingestion, injection, or absorption through the skin $[1,4,7,8]$. The difference lies in how it is absorbed, the
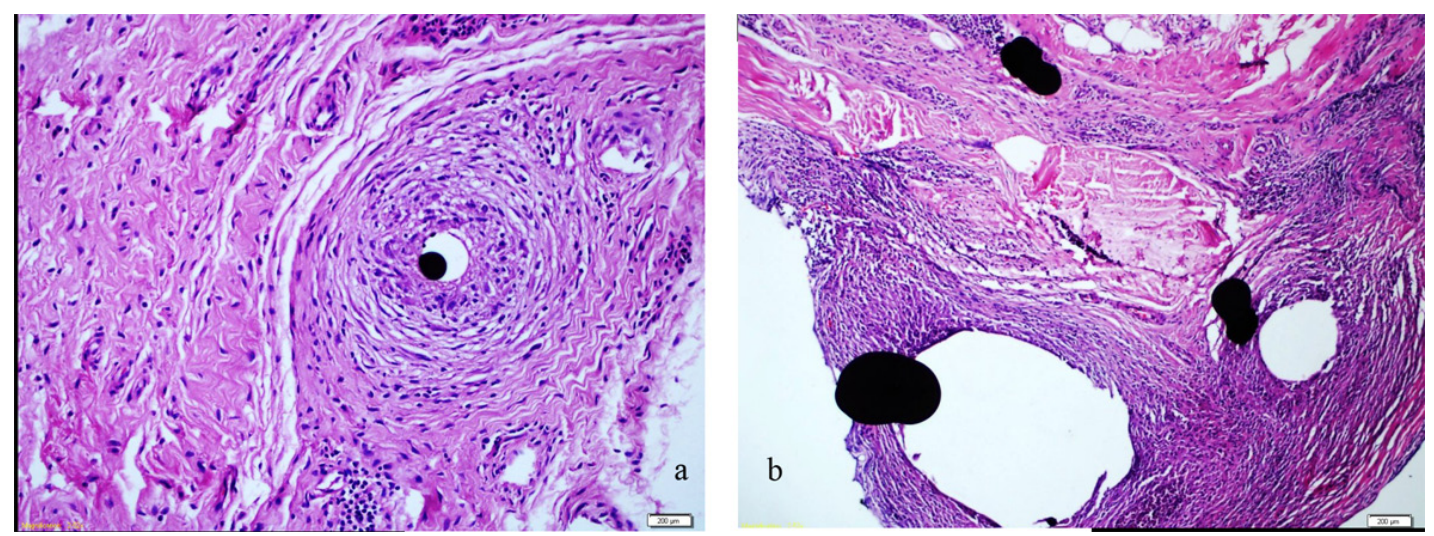

Figure 4. a: Histolopathological examination of the removed tissue on light microscopy: Mercury appears as spherical to eggshaped black pigmented globules and a mixed inflammatory cellular infiltrate composed of neutrophils, lymphocytes, histiocytes and fibrosis of chronic inflammation. $\mathrm{H}$ and $\mathrm{E} \times 440$. b: Histolopathological examination of the removed tissue on light microscopy: Mercury appears as spherical to egg-shaped black pigmented globules and a mixed inflammatory cellular infiltrate composed of neutrophils, lymphocytes, histiocytes and fibrosis of chronic inflammation. H and E x 220. 
clinical signs and symptoms, and the response to treatment modalities. Neurologic, gastrointestinal, and renal systems are the most commonly affected organ systems in mercury exposure [1, 4, 5, 7-10]. Elemental mercury $(\mathrm{Hg})$ is found in liquid form including mercury in thermometers and vaporizes at room temperature $[5,8,10]$. Elemental mercury is not well absorbed by the GI tract, when ingested (eg. thermometers) is only mildly toxic [1, 2, 4-7], and absorption through the skin is limited unless tissue inflammation is present or the mercury is retained over a prolonged period of time [5]. After absorption, elementary mercury is gradually oxidized into soluble mercuric salts that bind sulfhydryl groups, resulting in enzyme inhibition and pathologic cell membrane alteration. This can lead to systemic toxicity particularly in the central nervous system and the kidneys $[1,5,6,9]$. Mercury is mainly excreted from the body by the kidneys, but the rate of excretion is usually very slow [1], like in our patient, and traces of mercury can be seen on radiographs, and in urine even two years after the index event [7]. Measurements of mercury in blood and urine are useful in quantifying the degree of exposure. Clinically significant poisoning with mercury is unlikely if blood and urine concentrations are below $100 \mu \mathrm{g} / \mathrm{L}[1,6]$. Although in two different measurements the patient had urine mercury levels above the mentioned range, finding a measurable amount of mercury in blood and urine does not mean that the level of mercury causes an adverse health effect [1]. Individual prognosis is dependent on the severity of exposure, the form of the mercury compound, the route of administration, and the duration of exposure $[1,6,9,10]$ In cases of mercury poisoning, close monitoring of the clinical status of the patient is required with follow-up for at least two years [7].

Complete recovery is possible when the exposure to inorganic (i.e. elemental, mercuric salt) and organic compounds is mild. Treatment with chelators should be considered in patients with acute symptoms arising from the central nervous system due to confirmed mercury poisoning $[6,8]$. There is no proof that chelation therapy induces significant removal of subcutaneous mercury, and even in patients demonstrating increased elimination, clinical benefit may not be apparent [8]. As recommended [2, 5, 8, 9], surgical excision of the mercury granulomas lowered serum and urine mercury levels in this case. There is still some metallic mercury left in the soft tissues of the hand, and the patient will be followed for the ongoing absorption which may unexpectedly cause systemic lesions.

\section{Acknowledgement}

We would like to thank Ankara Duzen Laboratory for measuring mercury concentrations in the blood and urine in their laboratory.

\section{Authors' Contributions}

$\mathrm{DE}$ and ARS acquired the data and wrote the manuscript, MT and CA did the operation and handled the specimen to the Pathology Laboratory, ET, EK, YCK, SHT and MP performed the clinical follow-up of the patient, SB performed the pathological evaluation.

\section{Informed Consent}

Written informed consent was obtained from the patient for publication of this case report and any accompanying images.

\section{Competing Interests}

The authors declare that they have no competing interests.

\section{References}

1. Centers for Disease Control and Prevention (CDC). Fourth National Report on Human Exposure to Environmental Chemicals. Mercury CAS No. 7439-97-6, 2009; p: 218-26.

2. Ellabban MG, Ali R, Hart NB. Subcutaneous metallic mercury injection of the hand. Br J Plast Surg. 2003;56(1):47-49.

3. Chitkara R, Seriff NS, Kinas HY. Intravenous selfadministration of metallic mercury in attempted suicide. Report of a case with serial roentgenographic and physiologic studies over an 18-month period. Chest. 1978;73(2):234-236.

4. Diner BM. Mercury toxicity in emergency medicine. Available from: http://emedicine.medscape.com/ article/819872-overview. [accessed on 2009 Sep $18]$.

5. Vano-Galvan S, Jimenez-Garcia D, Vano-Galvan E, Jaen P. Accidental subcutaneous implant of mercury after thermometer trauma. J Am Acad Dermatol. 2009;61(3):535-537.

6. Elinder CG.Epidemiology and toxicity of mercury. Available from: http://www.uptodate.com/contents/epidemiology-and-toxicity-of-mercury. [accessed on 2011 June 15].

7. Wale J, Yadav PK, Garg S. Elemental mercury poisoning caused by subcutaneous and intravenous injection: An unusual self-injury. Indian J Radiol Imaging. 2010;20(2):147-149.

8. Kayias EH, Drosos GI, Hapsas D, Anagnostopoulou GA. Elemental mercury-induced subcutaneous granuloma. A case report and review of the literature. Acta 
Orthop Belg. 2003;69(3):280-284.

9. Haghdoost M, Poorhasan A, Ghabili K, Mashrabi O.Subcutaneous injection of metallic mercury mimicking bacterial cellulities:A case report. Am J Infect Dis
2010; 6:54-6.

10. Yildirim R, Erdem F, Gundogdu M, Bilen Y, Koca E, Yillikoglu Y, Sahin YN. Mercury toxicity:A family case report. Turk J Hematol 2012; 29:76-9. 